Revista Complutense de Educación

ISSNe: 1988-2793

http://dx.doi.org/10.5209/RCED.56016

\title{
Educación para la salud y prevención de riesgos psicosociales en adolescentes
} y jóvenes

Autoras: Juan.A. Morón Marchena, Itahisa Pérez Pérez y Encarnación Pedrero García (Coords.) Editorial: Edicions UIB. Año de publicación: 2017

Número de páginas: 271

ISBN: 978-84-277-2221-7

Educación para la salud y prevención de riesgos psicosociales en adolescentes y jóvenes, coordinado por Juan Agustín Morón Marchena, Itahisa Pérez-Pérez y Encarnación Pedrero García, profesores de la Universidad Pablo Olavide de Sevilla, que a su vez, cuenta con la colaboración de varios autores expertos en la materia, es un libro fundamental dirigido a un público muy heterogéneo (profesores, padres, familias, tutores...) que nos permite comprender y reflexionar, a lo largo de 12 capítulos, sobre la importancia de la promoción de la salud, el conocimiento de conductas de riesgo y sus efectos, junto con la necesidad de participación en el propio proceso de autonomía y empoderamiento de un colectivo que debido a sus características puede llegar a ser considerado como de riesgo.

La adolescencia es una etapa compleja, de cambios constantes y eso precisamente es lo que nos vamos a encontrar en el primer capítulo (Gonzalo Musitu Ochoa y Belén Martínez Ferrer), una introducción a las conductas disruptivas en la adolescencia, donde se hace especial hincapié en la violencia y el comportamiento delincuencial, abordando la importancia de la familia y la escuela, como agentes fundamentales a la hora de trabajar activamente la prevención.

A su vez, e íntimamente relacionado con el párrafo anterior, nos topamos con el segundo capítulo (Miguel Ángel Rodríguez Felipe), que aborda, los considerados como comportamientos de riesgo con el objetivo de favorecer su prevención, apoyado en los principales modelos que nos permiten comprender desde una perspectiva teórica las conductas de riesgo y que tiene como núcleo central la Teoría del Aprendizaje Social de Bandura. Todo ello, es completado con información relevante sobre los distintos ámbitos de intervención preventiva en drogas, teniendo en cuenta los espacios y contextos donde puede ser desarrollado.

El conocimiento de la intervención socioeducativa y sus áreas prioritarias, resulta fundamental para poder intervenir con el rigor adecuado, adquiriendo mayor notoriedad en un campo muy concreto y necesario como es la educación para la salud. El capítulo tercero (Rosa $\mathrm{M}^{\mathrm{a}}$ Macarro Carballar), nos presenta los distintos ámbitos de intervención en materia de prevención de las conductas de riesgo (plan, programa, proyecto, actividad y tarea), modelos concretos que nos permiten llegar al aprendizaje significativo, junto con la importancia de los valores prosociales como medio principal para reducir la conducta de riesgo, sin olvidarnos de la participación social e implicación de la persona en el propio proceso formativo para evitar situaciones de exclusión. 
El conocimiento de sustancias y los efectos sobre su consumo, es un aspecto fundamental a trabajar desde la educación para la salud, más si cabe, cuando intervenimos con colectivos especialmente vulnerables como son los adolescentes y jóvenes. Precisamente el capítulo cuarto (Celia Prat Pérez), nos permite comprender la existencia y características de determinadas sustancias, estereotipos respecto a su consumo y los principales efectos que puede tener en nuestro organismo, ofreciendo de esta manera, una perspectiva realista sobre dicho fenómeno, sirviendo de base para orientar adecuadamente los programas dirigidos a la prevención.

Seguimos avanzando en este apasionante manual y llegamos al capítulo quinto (Alfonso Borrego Prieto), donde se aborda el fenómeno del consumo de drogas por parte de los adolescentes y jóvenes, la percepción social del problema y la etiopatogenia y función social, que nos permite comprender con mayor detalle las causas del consumo, los objetivos de dicho consumo y la influencia de determinados factores (características individuales, ambiente familiar, ambiente escolar, grupo de iguales, ambiente macrosocial...) que pueden ser decisivos a la hora de incrementar la probabilidad de inicio en el consumo y que éste genere problemas de mayor envergadura.

Por otra parte, cuando hablamos de adicción, no solemos pensar en algo tan importante y necesario actualmente como son las nuevas tecnologías, insertadas en una sociedad caracterizada por la información y el conocimiento. Pero la realidad es otra distinta y eso es precisamente lo que nos encontramos en el capítulo sexto (Luisa Torres Barzabal, José Manuel Hermosilla Rodríguez, e Irene Benítez Hernández de Santaolalla), encargado de aportar una visión objetiva y documentada sobre las variables a considerar en la evaluación de posibles adicciones a las TIC, divididas a su vez en diferentes categorías que nos permitirán una mejor estructuración a la hora de valorar determinadas situaciones nocivas que pueden conllevar riesgos cada vez más heterogéneos.

El acoso escolar, es otra de las realidades presente en multitud de centros educativos y que a su vez, muchos adolescentes sufren diariamente. Pero además, las nuevas tecnologías a las que hicimos hincapié en el párrafo anterior, también están encontrando su lugar a la hora de ejercer dicho acoso. Un ejemplo de ello, es precisamente el ciberacoso, elemento central del capítulo séptimo (María Teresa Salces Rodrigo) y que nos introduce en sus características principales, las diferentes modalidades en las que podemos encontrar este ciberbulling, los factores influyentes y efectos generados tanto en la víctima como en el agresor o las familias. A su vez, nos presenta una interesante propuesta de intervención, centrada en el incremento de las medidas preventivas para su detección, control y futura erradicación desde el contexto educativo.

La sociedad en la que vivimos e interactuamos actualmente, ha generado un aumento considerable del consumo sin la necesidad de que esté presente una sustancia concreta, derivado del aumento de la oferta y recursos a nuestro alcance. Una realidad de estas características, también implica comportamientos adictivos, lo que en el octavo capítulo (Almudena Martínez Gimeno, $M^{a}$ Carmen Muñoz Díaz y David Cobos Sanchiz) se conoce como socioadicciones o adicciones sin sustancia, donde se hace un completo análisis de la concepción de adicción (uso, abuso y dependencia), se abordan la principales teorías explicativas de dicho fenómeno, factores de riesgo y consecuencias de las socioadicciones junto con un interesante análisis de los entornos principales (macrosocial y microsocial) y factores de protección que favorecen la integración.

La práctica sexual y la sexualidad en general, es un aspecto fundamental que debe ser abordado con adolescentes y jóvenes, y más, teniendo en cuenta las carac- 
terísticas de una etapa, en donde la experimentación es un elemento diferenciador. El noveno capítulo (Encarnación Pedrero García), nos ofrece un detallado análisis, compuesto de estudios relevantes para justificar la importancia de una educación sexual, donde se elimine la prohibición y el peligro de los programas desarrollados en muchos centros educativos y de esta manera aportar una visión racional y aperturista de la sexualidad, abordado desde múltiples contextos (escuela, universidad, asociaciones, centros juveniles...).

Asimismo, resulta necesario relacionar esta información relativa a la educación sexual, con los contenidos abordados en el décimo capítulo (J. Carlos GonzálezFaraco, C. M ${ }^{\mathrm{a}}$ Aránzazu Cejudo Cortés e Inmaculada Iglesias-Villarán), centrado en el VIH en jóvenes, una realidad, todavía desconocida y altamente estigmatizada. Por todo ello, aportamos tanta validez a la información expuesta en dicho capítulo ya que nos permitirá, ampliar nuestro conocimiento sobre los riesgos asociados a la enfermedad y vislumbrar relatos vitales para conocer con mayor precisión las percepciones personales, miedos, incertidumbres de jóvenes afectados con edades comprendidas entre 25 y 33 años.

Una vez vistos algunos de los principales aspectos relativos a la educación para la salud y prevención de riesgos psicosociales, nos encontramos con el onceavo capítulo (Fernando López Noguero), donde se profundiza en una propuesta de intervención muy concreta como es la animación sociocultural, ocio y tiempo libre con adolescentes y jóvenes, presentándose como un instrumento y alternativa para conseguir un proceso de empoderamiento que tenga en cuenta los intereses, motivaciones y participación del sujeto en su propio desarrollo y crecimiento personal.

Por último y para poder articular todo lo resaltado en líneas anteriores, no podemos olvidarnos de algo tan importante como la planificación y elaboración de proyectos socioeducativos. El doceavo capítulo (Itahisa Pérez-Pérez, Alfredo Jesús Castillo Manzano y Juan Agustín Morón Marchen), encargado de finalizar el manual, nos permite conocer con mayor detalle y a través de aspectos teórico-prácticos, elementos tan importantes como la planificación, sus elementos previos y el diseño de programas y proyectos para estructurar la intervención socioeducativa y sociocomunitaria con adolescentes y jóvenes, que nos permitirá abordar con rigor, cualquier intervención en esta área. 